\title{
OPEN Evaluation on soil fertility quality under biochar combined with nitrogen reduction
}

\author{
Xiaoqin Tian ${ }^{1,2}$, Zhuo $\mathrm{Li}^{2}$, Yifan Wang ${ }^{1},{\text { Biao } \mathrm{Li}^{1} \text { \& Longchang Wang }}^{1 凶}$
}

A two-year consecutive field experiment was conducted in purple soil in southwest China, to clarify the effects of biochar (0, 10, 20 and $40 \mathrm{t} \mathrm{ha}^{-1}$, namely, B0, B10, B20 and B40) combined with nitrogen reduction $(100 \%, 80 \%$ and $60 \%$ of conventional nitrogen application rate, namely, N100, N80 and $\mathrm{N60}$ ) on soil fertility. The performance of thirty-four indices related to soil chemical, physical and biological properties was evaluated by gray correlation analysis, principal component analysis and cluster analysis to determine the most appropriate mode for soil fertilization, and to identify the main soil environmental factors affecting rapeseed yield under the biochar combined with nitrogen reduction. The results indicated that available phosphorus, geometric mean diameter of water stability, fungi number, and the utilization of sugars, amino acids, polymers and carboxylic acids by microorganisms could be used as the main soil factors affecting rapeseed yield. The highest score of soil quality was observed in N100B10 treatment, followed by N80B10 and N100B20 treatments, which were almost in line with the results of rapeseed yields. Cluster analysis classified 12 treatments into 5 main groups on the basis of the measured parameters, which was mostly consistent with the result of

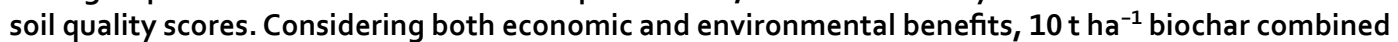
with $144 \mathrm{~kg} \mathrm{ha}^{-1}$ nitrogen was the best combination to restore crop productivity and soil quality, and to achieve nitrogen decreasing and benefit increasing. This study provided scientific basis for the rational fertilization and scientific management of biochar combined with nitrogen fertilizer in purple soil area of southwest China.

Soil fertility is the core of soil quality as well as the foundation of sustainable agricultural development, which plays an important role in maintaining soil quality and ensuring the sustainable use of soil resources ${ }^{1}$. In recent years, due to unreasonable fertilization management measures, especially over-reliance on nitrogen fertilizers in southwest China as main rapeseed producing area in $\mathrm{China}^{2}$, has caused many problems such as soil acidification, greenhouse gas emissions, groundwater nitrate pollution, organic matter content declining and production efficiency decreasing and so on ${ }^{3-6}$, which has become a serious threat to the sustainable development of agriculture in southwest China. Therefore, it is very urgent to explore economic and effective fertilization measures for achieving the sustainable development of agriculture.

Biochar is widely used in soil improvement as well as crop yield increase because of its unique structure and properties (such as abundant pores and large specific surface area) ${ }^{7-10}$. Liu et al. ${ }^{11}$ and Wang et al..$^{12}$ showed that biochar significantly enhanced the soil quality, and crops yield and quality for the improvement of soil $\mathrm{pH}$, activated carbon component, soil aggregation, total soil porosity and soil microbial biomass. Besides, the appropriate amount of nitrogen fertilizer plays an important role in increasing crop yields and soil quality. Nabilla et al. ${ }^{13}$ proved that urea $\left(300 \mathrm{~kg} \mathrm{ha}^{-1}\right)$ gave the best black rice yield with high 1000-grain weight and high soil fertility. A number of previous studies also have proved that biochar combined with chemical fertilizer could reduce the leaching of soil nutrients, delay the release of nutrients, thereby increasing crop yields and improving fertilizer utilization ${ }^{14-16}$. Peng et al. ${ }^{17}$ suggested that biochar combined with chemical fertilizers could increase $\mathrm{C}$ stability and $\mathrm{N}$ retention in soil and improve $\mathrm{N}$ uptake by maize, while the loss of $\mathrm{N}$ was minimized. Moreover, Zaid et al. ${ }^{18}$ implied that within the appropriate nitrogen application range, the interaction of biochar and nitrogen might had an economical approach towards better utilization of nitrogen and sustainable crop production. However, because of the complexity of biochar, soil and crop characteristics and types, no a unanimous conclusion about biochar in soil improvement and crop productivity increasing. Zahra et al. ${ }^{19}$ indicated that there was an increase

${ }^{1}$ College of Agronomy and Biotechnology, Southwest University, Chongqing 400715, China. ${ }^{2}$ Crop Research Institute, Sichuan Academy of Agriculture Sciences, Chengdu 610066, Sichuan, China. ${ }^{\square}$ email: wanglc@ swu.edu.cn 
of $46.29 \%$ and $13.4 \%$ in grain yield and biological yield, respectively, due to the an increase of soil organic carbon in treatment containing biochar and compost over untreated control. However, Kalu et al..$^{20}$ found that added biochars had minor long-term effects on the crop biomass yield, plant nutrient contents and plant nutrient uptake in both soil types (nutrient-poor, coarse textured Umbrisol and fertile, fine-textured Stagnosol). Therefore, biochar application in agricultural production should adapt to local conditions and be applied reasonably according to soil and crop types.

Purple soil is a unique and main soil type in southwest China. It is generally rich in calcium and nutrients, but is easily eroded and weathered for the shallow soil layer, so its physical and chemical properties, and biological characteristics are different from other varieties of soil ${ }^{21}$. Therefore, it is urgent to objectively and comprehensively evaluate the impact of biochar combined with nitrogen reduction on soil fertility, to find out the main soil environmental factors affecting rapeseed yield and to seek for the best combination ratio between biochar and nitrogen, so as to improve soil quality while improving economic benefits. Grey correlation degree, principal component analysis and cluster analysis are often used as statistical methods to assess soil fertility ${ }^{22-23}$. So far, there are few reports to comprehensively evaluate the effect of biochar combined with nitrogen reduction on purple soil quality from the physical, chemical and biological perspectives by the statistical methods. The aims of this study were to: (1) determine which soil environmental factors are the most important indexes affecting rapeseed yield under biochar combined with nitrogen reduction, and (2) identify the soil fertilization effect of biochar combined with nitrogen reduction to explore their best combined application amount. Results of this study will provide a scientific basis for the ration fertilization and scientific management of the combined application of biochar and nitrogen fertilizer in purple soil in southwest China.

\section{Materials and methods}

Research area. The study was conducted in the Yunyang Experimental Station $\left(108^{\circ} 54^{\prime} \mathrm{E}, 30^{\circ} 55^{\prime} \mathrm{N}\right.$; altitude of $700 \mathrm{~m}$ ), Southwest University, Chongqing, China. The study area has a subtropical monsoon humid climate with an average annual sunshine duration of $1500 \mathrm{~h}$, average annual temperature of $18.4^{\circ} \mathrm{C}$ average annual rainfall of $1100.1 \mathrm{~mm}$, and the rain period predominantly prolongs from June to September. Local soil type is clay loam in texture and Dystric Purple-Udic Cambosols according to the Chinese Soil Taxonomy (CRGCST 2001). Basic properties of $0-20 \mathrm{~cm}$ soil layer were as follows: $\mathrm{pH} 7.29$, total $\mathrm{N} 0.94 \mathrm{~g} \mathrm{~kg}^{-1}$, total C $7.14 \mathrm{~g} \mathrm{~kg}^{-1}$, available $\mathrm{N} 37.45 \mathrm{mg} \mathrm{kg}^{-1}$, available $\mathrm{P} 2.36 \mathrm{mg} \mathrm{kg}^{-1}$, and available $\mathrm{K} 72.58 \mathrm{mg} \mathrm{kg}^{-1}$, respectively.

The tested biochar was purchased from the Nanjing Qinfeng Straw Technology Co., Ltd. (Nanjing, China), which was made by pyrolysis of the rice (Oryza sativa L.) straw with limited oxygen supply at $500{ }^{\circ} \mathrm{C}$ for $2 \mathrm{~h}$. Its properties were as follows: total $\mathrm{N} 0.61 \mathrm{~g} \mathrm{~kg}^{-1}$, total P $1.99 \mathrm{~g} \mathrm{~kg}^{-1}$, total $\mathrm{K} 27.15 \mathrm{~g} \mathrm{~kg}^{-1}$, total C $537.97 \mathrm{~g} \mathrm{~kg}^{-1}$ and $\mathrm{pH} 8.70$.

Experimental design. A two-year filed experiment (2017-2019) was performed in a completely randomized design with twelve treatments in triplicates including two factors. The first factor was the application of biochar including B0 $\left(0 \mathrm{tha}^{-1}\right), \mathrm{B} 10\left(10 \mathrm{tha}^{-1}\right), \mathrm{B} 20\left(20 \mathrm{tha}^{-1}\right)$ and B40 $\left(40 \mathrm{t} \mathrm{ha}^{-1}\right)$; and the second factor is the application level $\mathrm{N}$ fertilizer including conventional rate (application amount by local farmers)-180 $\mathrm{kg} \mathrm{N} \mathrm{ha}^{-1}$ (N100), $80 \%$ of conventional rate-144 $\mathrm{kg} \mathrm{N} \mathrm{ha}^{-1}$ (N80) and $60 \%$ of conventional rate-108 $\mathrm{kg} \mathrm{N} \mathrm{ha}^{-1}$ (N60). The plot size was $3 \mathrm{~m} \times 6 \mathrm{~m}$ with a border $(0.5 \mathrm{~m}$ wide) between plots. Biochar was applied to soil only in the first year before the sowing of rapeseed. Each treatment plot received the same amount of potassium $\left(90 \mathrm{~kg} \mathrm{~K}_{2} \mathrm{O} \mathrm{ha}^{-1}\right)$ and phosphorus $\left(90 \mathrm{~kg} \mathrm{P}_{2} \mathrm{O}_{5} \mathrm{ha}^{-1}\right)$. Further details of fertilizer application have been reported by Tian et al. ${ }^{24}$, being the same for the two-year experiment. Weed, pesticide, and pest management kept the same with the local farmers' rapeseed management practices. Winter rapeseed (Sanxiayou No.5) was used in the experiment, which was sowed on 21 October 2017 and on 16 October 2018, respectively, and was harvested on 1 May in both years (2018 and 2019).

Sampling and analysis of soil and crop. Crop yield. Rapeseed was hand-harvested when 70-80\% of total seeds changed their color from green to black on 1 May 2019, and each plot was separately harvested for seed yield. Seed yield was calculated using $6 \%$ as standard seed moisture content.

Soil indices. After the rapeseed harvest, soil samples were collected from all plots. Five sampling points were randomly selected within each plot. At each point, twenty soil cores of $2.5 \mathrm{~cm}$ diameter and $20.0 \mathrm{~cm}$ depth were taken in a $1 \mathrm{~m}$ radius of the point. All soil cores from each point were put in a plastic bag and thoroughly bulked, crumbled and mixed for physical, chemical and biological analyses. By dividing each soil sample into two subsamples, one subsample was ground, passed through a $2-\mathrm{mm}$ sieve and was air-dried for the analyses of soil organic matter (SOM), total nitrogen (TN), total phosphorus (TP), total potassium (TK), alkali-hydrolyzale nitrogen (AN), available phosphorus (AP), available potassium $(\mathrm{AK})^{25}$, particulate organic carbon (POC), watersoluble organic carbon (DOC), easily oxidized organic carbon (AOC) $)^{26}$, sucrase (SUC) and urease (URE) ${ }^{27}$, and another one was ground, passed through a 2-mm sieve and was stored in a refrigerator at $-20^{\circ} \mathrm{C}$ for the analyses of structural and functional characteristics of soil microbial community ${ }^{28}$. At the same time, mixed soil samples $(0-20 \mathrm{~cm})$ from five points in each plot were taken using a shovel for soil aggregates analyses ${ }^{24}$.

Drying method was used to determine soil water content (SWC); soil temperature (ST) was measured by temperature probe on the LI6400-09 (LI-COR Inc., Lincoln, NE); potassium dichromate oxidation method was used to determine SOM and DOC content; TN was measured by the Kjeldahl method; TP was determined by Mo-Sb colorimetric method; $\mathrm{TK}$ was determined by $\mathrm{NaOH}$ melting and analyzed using an atomic spectrophotometry; AN was determined by diffusion-absorption method; AP was quantified by colorimetric analysis following extraction of soil with $0.5 \mathrm{~mol} \mathrm{~L}^{-1} \mathrm{NaHCO}_{3}$; AK was measured using $1.0 \mathrm{~mol} \mathrm{~L}^{-1} \mathrm{CH}_{3} \mathrm{COONH}_{4}$ extraction; POC 
was determined by sodium hexametaphosphate dispersion method; AOC was measured by potassium permanganate oxidation method; SUC was measured by 3,5-dinitrosalicylic acid colorimetric determination method; URE was measured by phenol-sodium hypochlorite indophenol colorimetry method; amount of bacteria (B), fungi (F), actinomycetes (A), gram-positive bacteria (GP), gram-negative bacteria (GN) was measured by the Bligh-Dyer method; utilization of sugars (S), amino acids (AA), phenolic acids (PA), carboxylic acids (CA), amines (AM) and polymers $(\mathrm{P})$ by microorganism was measured using commercial Biolog EcoPlate (Biolog Inc., CA, USA).

Shannon index $(\mathrm{H})$, Simpson index (D), and evenness index (E) were calculated by the following equations:

$$
\begin{gathered}
\mathrm{AWCD}=\sum\left(C_{i}-R_{i}\right) / n \\
\mathrm{H}=-\sum P_{i}\left(\ln P_{i}\right) \quad P_{i}=\left(C_{i}-R_{i}\right) / \sum\left(C_{i}-R_{i}\right) \\
\mathrm{D}=1-\sum P_{i}^{2} \\
\mathrm{E}=\mathrm{H} / \ln \mathrm{S}
\end{gathered}
$$

where $n$ is the 31 carbon sources on the ECO board; $C_{i}$ and $R_{i}$ and are the optical density values of the microwell and the control well respectively; $P_{i}$ is the ratio of the absorbance of a particular well $i$ to the sums of absorbance of all 31 well at $120 \mathrm{~h} ; \mathrm{S}$ is the number of color change holes, which represents the number of carbon source used by the microbial community; Average well color development (AWCD), representing the overall carbon substrate utilization potential of cultural microbial communities across all wells per plate.

In order to investigate the aggregate structure, all bulk clod samples from each plot were carefully mixed and then gently sieved to pass through a 10-mm sieve. According to the wet-sieving and dry-sieving protocol, the tested soil was fractionated into $>5,2 \sim 5,1 \sim 2,0.25 \sim 1$ and $<0.25 \mathrm{~mm}$ aggregates, respectively. All separated aggregates were dried in oven at $60^{\circ} \mathrm{C}$ for determining their properties. Macroaggregate content (R), average weight diameter (MWD) and geometric mean diameter (GMD) were calculated by the following equations:

$$
\begin{gathered}
\mathrm{DR}_{0.25}=\left(\mathrm{WR}_{0.25}\right)=\frac{\sum_{i=1}^{n}\left(w_{i}>0.25\right)}{\sum_{i=1}^{n}\left(w_{i}\right)} \times 100 \% \\
\mathrm{D}-\mathrm{MWD}(\mathrm{W}-\mathrm{MWD})=\sum_{i=1}^{n}\left(\bar{d}_{i} w_{i}\right) \\
\mathrm{D}-\mathrm{GMD}(\mathrm{W}-\mathrm{GMD})=\exp \left[\frac{\sum_{i=1}^{n} m_{i} \ln \bar{d}_{i}}{\sum_{i=1}^{n} m_{i}}\right]
\end{gathered}
$$

where $\mathrm{DR}_{0.25}$ and $\mathrm{WR}_{0.25}$ are the proportion of $>0.25 \mathrm{~mm}$ soil mechanical-stable aggregates and water-stable aggregates, respectively; D-MWD and W-MWD are the mean weight diameter of mechanical-stable aggregates and water-stable aggregates $(\mathrm{mm})$, respectively; D-GMD and $\mathrm{W}-\mathrm{GMD}$ are the mean geometric diameter of mechanical-stable aggregates and water-stable aggregates $(\mathrm{mm})$, respectively; $m_{i}$ is mass in size fraction $i$; and $w_{i}$ is the proportion (\%) of the total sample mass in size fraction $i$ and $d_{i}$ is mean diameter of size fraction $i$.

Evaluation of soil fertility. Grey correlation analysis. Grey correlation analysis refers to a method of quantitative description and comparison of a system's development and change. The basic idea is to determine whether they are closely connected by determining the geometric similarity of the reference data column and several comparison data columns, which reflects the degree of correlation between the curves ${ }^{29}$. The grey relational coefficient $\xi_{\mathrm{i}}(k)$ can be expressed as follows:

$$
\begin{gathered}
\xi(k)=\frac{\min _{i} \min _{k}\left|x_{0}(k)-x_{i}(k)\right|+\rho \max _{i} \max _{k}\left|x_{0}(k)-x_{i}(k)\right|}{\left|x_{0}(k)-x_{i}(k)\right|+\rho \max _{i} \max _{k}\left|x_{0}(k)-x_{i}(k)\right|} \\
x_{i}^{k}=\frac{x_{i}^{k}}{\max _{i} x_{i}^{k}} \\
\gamma_{i}=\frac{1}{n} \sum_{k=i}^{n} \xi_{i}(k)
\end{gathered}
$$




$$
\begin{gathered}
\omega_{i(\gamma)}=\frac{1}{n} \sum_{i=1}^{n} \gamma_{i} \\
G_{i}^{k}=\sum_{i=1}^{n}\left(\xi_{i} \times \omega_{i(\gamma)}\right), \quad k=1,2,3, \ldots, n ; \quad i=1,2,3, \ldots, n
\end{gathered}
$$

where $x_{i}^{k}$ The $i$ trait observation value of treatment $k$; $\max _{i} x_{i}^{k}$ The maximum value of the $i$ trait in all treatments; $\min _{i} x_{i}^{k}$ The minimum value of the $i$ trait in all treatments; min min $\left|x_{0}(k)-x_{i}(k)\right|$ Second level minimum difference; $\max _{i} \max _{k}\left|x_{0}(k)-x_{i}(k)\right|$ Second level maximum difference; $\rho$ Resolution coefficient (0.5).

Principal component analysis. Principal component analysis refers to a multivariate statistical method that converts multiple indicators into several comprehensive indicators by the idea of dimensionality under the premise of losing little information. It simplifies the complexity in high-dimensional data while retaining trends and patterns $s^{30}$.

Cluster analysis. Cluster analysis comprises a range of methods for classifying multivariate data into subgroups. Using the euclidean distance as a measure of the difference in the fertility of each treatment, the shortest distance method was used to systematically cluster according to the degree of intimacy and similarity of soil fertility levels. By organizing multivariate data into such subgroups, clustering can help reveal the characteristics of any structure or patterns present ${ }^{31}$.

Statistical analysis. Correlation analysis was performed to assess the relationships between rapeseed yield and soil attributes. Grey correlation analysis and principal component analysis were performed to establish comprehensive score for soil fertility and the main soil factors affecting rapeseed yield. Cluster analysis was used to cluster the soil fertility of each treatment. All the statistical analyses were performed using Excel 2018 (Office Software, Inc., Beijing, China) and SPSS 17.0 (SPSS Inc., Chicago, Illinois, USA). The comparisons of treatment means were based on LSD test at the $P<0.05$ probability level.

Ethics statement. Identifies Southwest University that approved the collection of plant or seed specimens. Confirms that all methods were carried out in accordance with relevant guidelines and regulations.

\section{Results}

Selection of evaluation indexes for soil fertilization. To comprehensively and objectively evaluate the impact of biochar combined with nitrogen reduction on soil fertility, and identify the main soil environmental driving factors affecting the high yield of rapeseed, following the principles of representativeness, stability, and comparability, 34 indicators from three perspectives of soil physics, chemistry and biology, which represented the status of soil fertility were selected (Table 1$)^{22-23}$. It can be seen that there were significant differences in various indexes of different treatments (except for TN, TP, TK and AN), which could be used to distinguish the fertility effect of different treatments. Then, correlation analysis was performed between the 34 selected indicators and rapeseed yield, and 27 indicators $\left(x_{1 \sim} x_{27}\right)$ with significant correlations with yield were selected as evaluation indicators based on science and rationalization (Table 2).

Grey correlation analysis. Comparison of correlation degree. According to the correlation analysis principle in the grey system theory, the greater the correlation of the evaluation index, the closer it is to the reference index, that is, the greater the weight coefficient of evaluation index, the greater the impact on yield. As could be seen from Table 3, AP (weight coefficient 0.041) and W-GMD (weight coefficient 0.040) had the greatest impact on yield in physical index, and URE (weight coefficient 0.040 ) of soil chemical indicators had the greatest impact on yield. Besides, in biological index, F (weight coefficient 0.040), S (weight coefficient 0.040), AA (weight coefficient 0.040 ), $\mathrm{P}$ (weight coefficient 0.040 ) and CA (weight coefficient 0.040 ) by microorganisms had the greatest impact on yield. These indicators could be used as the main soil factors affecting rapeseed yield under biochar combined with nitrogen reduction.

Comprehensive evaluation and analysis of soil fertilization. Grey comprehensive evaluation values were enumerated in Table 4. According to the result, the variation trend of soil fertility under each treatment was consistent with the change trend of yield. Under the same nitrogen level, the comprehensive evaluation value of B10 was greater than that of B0, B20 and B40 treatment; under the same biochar level, the comprehensive evaluation value of N60 and N80 treatment is less than N100 treatment. Among all the treatments, N100B10 had the highest comprehensive evaluation value, ranking first, followed by N100B20 and N80B10, and N60B40 had the lowest comprehensive evaluation value. Then the conclusion was that B10 was conducive to soil fertilization and crop yield increase, which combined with N80 could achieve nitrogen reduction and efficiency increase, while nitrogen reduction (N60) was not conducive to soil fertilization and crop yield increase.

Principal component analysis. Correlation analysis between evaluation indicators. By analyzing related relationship of 27 indicators of soil physics, chemistry, and biology highly correlated with yield (Table 5), signifi- 


\begin{tabular}{|c|c|c|c|c|c|c|c|c|c|c|c|c|}
\hline Index & N100B0 & N100B10 & N100B20 & N100B40 & N80B0 & N80B10 & N80B20 & N80B40 & N60B0 & N60B10 & N60B20 & N60B40 \\
\hline ST & $19.11 b c$ & $19.65 a$ & 19.42abc & 19.39abc & $19.07 \mathrm{c}$ & $19.54 \mathrm{a}$ & 19.34abc & 19.31abc & $19.14 \mathrm{bc}$ & 19.51a & $19.46 \mathrm{ab}$ & 19.34abc \\
\hline SWC & $0.134 \mathrm{ab}$ & $0.143 a$ & $0.138 \mathrm{ab}$ & $0.136 \mathrm{ab}$ & $0.133 \mathrm{ab}$ & $0.143 a$ & $0.137 \mathrm{ab}$ & $0.134 \mathrm{ab}$ & $0.132 \mathrm{~b}$ & $0.143 a$ & $0.138 \mathrm{ab}$ & $0.134 \mathrm{ab}$ \\
\hline SOM & $14.31 \mathrm{~b}$ & $14.69 \mathrm{ab}$ & $15.06 \mathrm{ab}$ & $16.30 \mathrm{a}$ & $13.79 \mathrm{~b}$ & $14.36 \mathrm{~b}$ & $14.59 \mathrm{~b}$ & 15.18ab & $13.71 \mathrm{~b}$ & $14.16 \mathrm{~b}$ & $14.49 \mathrm{~b}$ & $15.01 \mathrm{ab}$ \\
\hline $\mathrm{TN}$ & $1.12 \mathrm{~cd}$ & $1.27 \mathrm{a}$ & $1.25 \mathrm{ab}$ & $1.23 \mathrm{abc}$ & $1.11 \mathrm{~cd}$ & $1.21 \mathrm{abc}$ & $1.15 \mathrm{abc}$ & $1.15 \mathrm{abc}$ & $1.02 \mathrm{~d}$ & $1.17 \mathrm{abc}$ & $1.14 \mathrm{bcd}$ & $1.12 \mathrm{~cd}$ \\
\hline $\mathrm{TP}$ & $0.61 \mathrm{a}$ & $0.65 \mathrm{a}$ & $0.65 \mathrm{a}$ & $0.64 \mathrm{a}$ & $0.60 \mathrm{a}$ & $0.65 a$ & $0.65 \mathrm{a}$ & $0.63 \mathrm{a}$ & $0.59 \mathrm{a}$ & $0.66 \mathrm{a}$ & $0.65 a$ & $0.65 \mathrm{a}$ \\
\hline TK & $18.23 \mathrm{a}$ & $20.38 \mathrm{a}$ & 19.81a & $19.49 \mathrm{a}$ & $18.16 \mathrm{a}$ & $20.48 \mathrm{a}$ & $19.55 a$ & $19.08 \mathrm{a}$ & $17.95 \mathrm{a}$ & $20.58 \mathrm{a}$ & $19.15 a$ & 18.99a \\
\hline AN & $42.00 \mathrm{a}$ & $44.10 \mathrm{a}$ & $43.00 \mathrm{a}$ & $42.77 \mathrm{a}$ & $41.80 \mathrm{a}$ & $43.07 \mathrm{a}$ & $42.40 \mathrm{a}$ & $42.30 \mathrm{a}$ & $41.67 \mathrm{a}$ & $42.90 \mathrm{a}$ & $42.07 \mathrm{a}$ & $42.00 \mathrm{a}$ \\
\hline $\mathrm{AP}$ & $12.48 \mathrm{bcd}$ & $15.66 \mathrm{a}$ & 14.79ab & 13.81abcd & $11.27 \mathrm{~cd}$ & $14.88 \mathrm{ab}$ & 13.57abcd & $11.57 \mathrm{~cd}$ & $10.91 \mathrm{~d}$ & $14.27 \mathrm{abc}$ & 12.85abcd & $11.33 \mathrm{~cd}$ \\
\hline $\mathrm{AK}$ & $80.58 c$ & $90.09 a$ & $83.51 b c$ & $83.00 \mathrm{bc}$ & $80.98 \mathrm{c}$ & $88.00 \mathrm{ab}$ & $83.06 \mathrm{bc}$ & $82.98 b \mathrm{bc}$ & $79.18 \mathrm{c}$ & 87.10ab & $83.92 b c$ & $82.96 \mathrm{bc}$ \\
\hline $\mathrm{DR}_{0.25}$ & 87.93ab & $91.38 \mathrm{a}$ & $87.55 \mathrm{ab}$ & $87.16 \mathrm{ab}$ & 87.83ab & $91.42 \mathrm{a}$ & $87.46 \mathrm{ab}$ & $87.01 \mathrm{ab}$ & $86.85 \mathrm{ab}$ & 91.11a & $85.38 \mathrm{~b}$ & $85.11 \mathrm{~b}$ \\
\hline $\mathrm{WR}_{0.25}$ & $66.05 \mathrm{~b}$ & $76.52 \mathrm{a}$ & 69.90ab & $68.42 \mathrm{~b}$ & $65.85 \mathrm{~b}$ & $75.68 \mathrm{a}$ & 69.63ab & 66.36b & $65.52 \mathrm{~b}$ & 70.47ab & $66.37 \mathrm{~b}$ & 65.93b \\
\hline D-MWD & $4.97 \mathrm{abc}$ & $5.09 a$ & $4.83 \mathrm{abc}$ & $4.86 \mathrm{abc}$ & $4.87 \mathrm{abc}$ & $5.04 \mathrm{abc}$ & $4.89 \mathrm{abc}$ & $4.83 \mathrm{abc}$ & $4.93 \mathrm{abc}$ & $5.08 \mathrm{ab}$ & $4.81 \mathrm{bc}$ & $4.77 \mathrm{c}$ \\
\hline W-MWD & $2.40 \mathrm{bc}$ & $2.81 \mathrm{a}$ & $2.66 \mathrm{abc}$ & $2.69 \mathrm{abc}$ & $2.42 \mathrm{bc}$ & $2.78 \mathrm{a}$ & $2.70 \mathrm{abc}$ & $2.69 \mathrm{abc}$ & $2.39 \mathrm{c}$ & $2.57 \mathrm{abc}$ & $2.65 \mathrm{abc}$ & $2.75 \mathrm{ab}$ \\
\hline D-GMD & $3.03 \mathrm{abc}$ & $3.38 \mathrm{a}$ & $2.91 \mathrm{abc}$ & $2.89 \mathrm{abc}$ & $2.96 \mathrm{abc}$ & $3.33 \mathrm{ab}$ & $2.92 \mathrm{abc}$ & $2.86 \mathrm{bc}$ & $2.95 \mathrm{abc}$ & $3.35 \mathrm{ab}$ & $2.76 \mathrm{c}$ & $2.71 \mathrm{c}$ \\
\hline W-GMD & $0.90 \mathrm{c}$ & $1.28 \mathrm{a}$ & $1.04 \mathrm{bc}$ & $1.04 \mathrm{bc}$ & $0.91 \mathrm{c}$ & $1.24 \mathrm{ab}$ & $1.06 \mathrm{bc}$ & $0.98 \mathrm{c}$ & $0.89 \mathrm{c}$ & $1.04 \mathrm{bc}$ & $0.98 \mathrm{c}$ & $0.99 \mathrm{c}$ \\
\hline POC & $2.90 \mathrm{cdef}$ & 3.38abcd & $3.46 \mathrm{abc}$ & $3.79 a$ & 2.88def & $3.11 \mathrm{bcdef}$ & $3.28 \mathrm{abcd}$ & $3.67 \mathrm{ab}$ & $2.57 \mathrm{f}$ & $2.70 \mathrm{ef}$ & 3.26abcde & $3.61 \mathrm{ab}$ \\
\hline DOC & $0.66 \mathrm{ab}$ & $0.75 a$ & $0.71 \mathrm{a}$ & $0.63 \mathrm{ab}$ & $0.66 \mathrm{ab}$ & $0.71 \mathrm{a}$ & $0.71 \mathrm{a}$ & $0.56 \mathrm{~b}$ & $0.64 \mathrm{ab}$ & $0.66 \mathrm{ab}$ & $0.62 \mathrm{ab}$ & $0.55 \mathrm{~b}$ \\
\hline $\mathrm{AOC}$ & $8.00 \mathrm{ab}$ & $8.80 \mathrm{a}$ & $7.50 \mathrm{bc}$ & $7.33 b c$ & $7.27 \mathrm{bc}$ & $7.50 \mathrm{bc}$ & $7.27 b c$ & $6.53 c$ & $6.77 \mathrm{c}$ & $7.30 \mathrm{bc}$ & $7.07 \mathrm{bc}$ & $6.30 \mathrm{c}$ \\
\hline SUC & $40.58 \mathrm{ab}$ & $41.90 \mathrm{a}$ & 39.47abcd & 38.61bcde & 39.44abcd & $39.70 \mathrm{abc}$ & 37.84bcde & $37.55 \mathrm{cde}$ & 38.55bcde & 39.58abcd & 36.91de & $36.39 \mathrm{e}$ \\
\hline URE & $2.12 \mathrm{bc}$ & $2.39 \mathrm{a}$ & $2.21 \mathrm{~b}$ & $2.05 \mathrm{~cd}$ & $1.51 \mathrm{fg}$ & $1.92 \mathrm{~d}$ & $1.73 \mathrm{e}$ & $1.64 \mathrm{ef}$ & $1.49 \mathrm{~g}$ & $1.76 \mathrm{e}$ & $1.65 \mathrm{ef}$ & $1.45 \mathrm{~g}$ \\
\hline B & $3.13 \mathrm{ab}$ & $3.86 a$ & $3.46 \mathrm{ab}$ & $2.98 \mathrm{ab}$ & $2.83 \mathrm{~b}$ & $3.94 \mathrm{a}$ & $3.43 \mathrm{ab}$ & $2.97 \mathrm{ab}$ & $2.82 \mathrm{~b}$ & $3.91 \mathrm{a}$ & $3.12 \mathrm{ab}$ & $2.63 \mathrm{~b}$ \\
\hline F & $0.41 \mathrm{abc}$ & $0.45 \mathrm{ab}$ & $0.41 \mathrm{abc}$ & $0.36 \mathrm{bcd}$ & $0.36 \mathrm{bcd}$ & $0.48 \mathrm{a}$ & $0.35 \mathrm{~cd}$ & $0.35 \mathrm{~cd}$ & $0.29 \mathrm{~d}$ & $0.41 \mathrm{abc}$ & $0.35 \mathrm{~cd}$ & $0.28 \mathrm{~d}$ \\
\hline A & $0.05 \mathrm{~cd}$ & $0.27 \mathrm{a}$ & $0.06 \mathrm{c}$ & $0.05 \mathrm{~cd}$ & $0.04 \mathrm{~cd}$ & $0.26 \mathrm{a}$ & $0.05 \mathrm{~cd}$ & $0.04 \mathrm{~d}$ & $0.04 \mathrm{~d}$ & $0.23 \mathrm{~b}$ & $0.04 \mathrm{~cd}$ & $0.03 \mathrm{~d}$ \\
\hline GP & $1.19 \mathrm{abc}$ & $1.27 \mathrm{ab}$ & $1.11 \mathrm{abc}$ & $1.04 \mathrm{bc}$ & $1.04 \mathrm{bc}$ & $1.43 \mathrm{a}$ & $1.22 \mathrm{abc}$ & $0.95 \mathrm{bc}$ & $1.00 \mathrm{bc}$ & $1.22 \mathrm{abc}$ & $1.07 \mathrm{bc}$ & $0.88 \mathrm{c}$ \\
\hline GN & $1.09 \mathrm{abc}$ & $1.32 \mathrm{ab}$ & $1.20 \mathrm{abc}$ & $1.05 b c$ & $1.00 \mathrm{bc}$ & $1.31 \mathrm{ab}$ & $1.29 \mathrm{ab}$ & $1.08 \mathrm{abc}$ & $1.04 \mathrm{bc}$ & $1.43 a$ & $1.06 \mathrm{bc}$ & $0.93 c$ \\
\hline S & $1.16 \mathrm{c}$ & $1.39 \mathrm{a}$ & $1.39 \mathrm{a}$ & $1.28 \mathrm{~b}$ & $1.09 \mathrm{~d}$ & $1.29 \mathrm{~b}$ & $1.17 \mathrm{c}$ & $1.15 \mathrm{c}$ & $1.08 \mathrm{~d}$ & $1.26 \mathrm{~b}$ & $1.17 \mathrm{c}$ & $1.13 \mathrm{~cd}$ \\
\hline AA & 1.10def & $1.38 \mathrm{a}$ & $1.37 \mathrm{a}$ & $1.13 \mathrm{cde}$ & $1.05 \mathrm{f}$. & $1.24 \mathrm{~b}$ & $1.15 \mathrm{~cd}$ & $1.07 \mathrm{ef}$ & $1.04 \mathrm{f}$. & $1.21 \mathrm{bc}$ & $1.14 \mathrm{cde}$ & $1.06 \mathrm{f}$ \\
\hline $\mathrm{PA}$ & $0.61 \mathrm{abc}$ & $0.65 a$ & $0.64 \mathrm{ab}$ & $0.62 \mathrm{abc}$ & $0.60 \mathrm{abc}$ & $0.64 \mathrm{ab}$ & $0.61 \mathrm{abc}$ & $0.58 \mathrm{~b}$ & $0.57 \mathrm{c}$ & $0.62 \mathrm{abc}$ & $0.60 \mathrm{abc}$ & $0.56 \mathrm{c}$ \\
\hline $\mathrm{CA}$ & $0.93 \mathrm{de}$ & $1.19 \mathrm{a}$ & $1.18 \mathrm{a}$ & $0.98 \mathrm{~cd}$ & $0.87 \mathrm{e}$ & $1.10 \mathrm{~b}$ & $1.01 \mathrm{c}$ & $0.92 \mathrm{de}$ & $0.87 \mathrm{e}$ & $1.06 \mathrm{bc}$ & $0.99 \mathrm{~cd}$ & $0.92 \mathrm{de}$ \\
\hline $\mathrm{AM}$ & $0.95 c$ & $1.07 \mathrm{ab}$ & $1.08 \mathrm{a}$ & $0.93 c$ & $0.88 \mathrm{c}$ & $0.97 \mathrm{bc}$ & $0.94 \mathrm{c}$ & $0.87 \mathrm{c}$ & $0.89 \mathrm{c}$ & $0.95 c$ & $0.93 c$ & $0.89 \mathrm{c}$ \\
\hline $\mathrm{P}$ & $1.02 \mathrm{ef}$ & $1.45 \mathrm{a}$ & $1.44 \mathrm{a}$ & $1.06 \mathrm{de}$ & $0.88 \mathrm{~g}$ & $1.34 \mathrm{~b}$ & $1.13 \mathrm{~cd}$ & $1.05 \mathrm{de}$ & $0.86 \mathrm{~g}$ & $1.16 \mathrm{c}$ & $1.00 \mathrm{ef}$ & $0.97 \mathrm{f}$ \\
\hline $\mathrm{H}$ & $3.314 \mathrm{bcd}$ & $3.339 a$ & $3.339 a$ & $3.304 d$ & $3.306 \mathrm{~d}$ & $3.341 \mathrm{a}$ & $3.325 \mathrm{abc}$ & $3.309 \mathrm{~cd}$ & $3.307 \mathrm{~d}$ & $3.329 \mathrm{ab}$ & $3.314 \mathrm{bcd}$ & $3.297 \mathrm{~d}$ \\
\hline $\mathrm{D}$ & $0.962 \mathrm{ab}$ & $0.963 a$ & $0.963 a$ & $0.961 \mathrm{~d}$ & $0.961 \mathrm{~cd}$ & $0.963 \mathrm{ab}$ & $0.962 b c$ & $0.961 \mathrm{~cd}$ & $0.961 \mathrm{~cd}$ & $0.962 \mathrm{ab}$ & $0.961 \mathrm{~cd}$ & $0.960 \mathrm{~d}$ \\
\hline E & $0.965 b c$ & $0.972 \mathrm{a}$ & $0.972 \mathrm{a}$ & $0.962 \mathrm{c}$ & $0.963 c$ & $0.973 \mathrm{a}$ & $0.968 \mathrm{ab}$ & $0.964 c$ & $0.963 c$ & $0.969 \mathrm{ab}$ & $0.965 b c$ & $0.960 \mathrm{c}$ \\
\hline
\end{tabular}

Table 1. Soil physical, chemical and biological indicators. ST $/{ }^{\circ} \mathrm{C}$; SWC $/ \%$; SOM, TN, TP, TK, POC, AOC/ $\mathrm{g} \cdot \mathrm{kg}^{-1}$; AN, AP, AK/mg.kg ${ }^{-1} \mathrm{DR}_{0.25}, \mathrm{WR}_{0.25} / \%$; DOC/g.L $\mathrm{L}^{-1}$; SUC, URE/mg.g ${ }^{-1} ; \mathrm{B}, \mathrm{F}, \mathrm{A}, \mathrm{GP}, \mathrm{GN} / \mathrm{nmol}^{-1} \mathrm{~g}^{-1}$.

cant or extremely significant correlation between the indicators was found, indicating that there was information overlap between indicators.

Calculation of feature vector. The feature values and contribution rates of each principal component were shown in Table 6 based on the descending dimension algorithm of 27 indicators (principal components with specified feature values greater than 1 were extracted). The eigenvalues of three principal components were greater than 1 with eigenvalues of $20.496,2.487$, and 1.708 , and contribution rates of $75.911 \%, 9.211 \%$, and $6.327 \%$, respectively. The cumulative contribution rate of the first three principal components reached $91.448 \%$, which could reflect enough information. Therefore, three main components were selected to comprehensively analyze and evaluate various indicators of soil environment under biochar combined with nitrogen reduction.

A component matrix (the data was not listed in this paper) could be obtained by the descending dimension algorithm, and feature vector could be calculated by each value in the component matrix (components 1,2 , and 3 ) dividing by the square root of the corresponding eigenvalue (Table 7). In the main component 1 , the variance of AP (feature vector 0.210 ) and W-GMD (feature vector 0.208 ) in the soil physical indicators, DOC (feature vector 0.181 ) in the soil chemical indicators, and B (feature vector 0.206), F (feature vector 0.201), S (feature vector 0.195), AA (feature vector 0.201), PA (feature vector 0.206), P (feature vector 0.204 ), CA (feature vector 0.203 ), $\mathrm{H}$ (feature vector 0.206 ), D (feature vector 0.204 ) and $\mathrm{E}$ (feature vector 0.205 ) in the soil biological indicators was the largest. Principal component 1 explained $75.911 \%$ of the difference, which represented most of the indicator information. Therefore, The indicator that contributed to the variance of principal component 1 could not only be used as the main factor affecting soil fertility but also be used as the main factor affecting rape yield under different treatments. 


\begin{tabular}{|c|c|c|c|c|c|}
\hline Soil physical index & Yield $\left(x_{0}\right)$ & Soil chemical index & Yield $\left(x_{0}\right)$ & Soil biological index & Yield $\left(x_{0}\right)$ \\
\hline ST & 0.285 & POC & 0.161 & $\mathrm{~B}\left(x_{15}\right)$ & $0.339^{*}$ \\
\hline $\operatorname{SWC}\left(x_{1}\right)$ & $0.330^{*}$ & $\operatorname{DOC}\left(x_{11}\right)$ & $0.452^{* *}$ & $\mathrm{~F}\left(x_{16}\right)$ & $0.527^{* *}$ \\
\hline SOM & 0.255 & $\operatorname{AOC}\left(x_{12}\right)$ & $0.527^{* *}$ & $\mathrm{~A}\left(x_{17}\right)$ & $0.484^{* *}$ \\
\hline $\mathrm{TN}\left(x_{2}\right)$ & $0.640^{* *}$ & $\operatorname{SUC}\left(x_{13}\right)$ & $0.542^{* *}$ & GP $\left(x_{18}\right)$ & $0.346^{*}$ \\
\hline $\mathrm{TP}$ & -0.001 & URE $\left(x_{14}\right)$ & $0.814^{* *}$ & GN & 0.269 \\
\hline TK & 0.318 & & & $\mathrm{~S}\left(x_{19}\right)$ & $0.797^{* *}$ \\
\hline $\mathrm{AN}\left(x_{3}\right)$ & $0.373^{*}$ & & & AA $\left(x_{20}\right)$ & $0.684^{* *}$ \\
\hline $\mathrm{AP}\left(x_{4}\right)$ & $0.573^{* *}$ & & & PA $\left(x_{21}\right)$ & $0.634^{* *}$ \\
\hline $\mathrm{AK}\left(x_{5}\right)$ & $0.433^{* *}$ & & & $\mathrm{CA}\left(x_{22}\right)$ & $0.658^{* *}$ \\
\hline $\mathrm{DR}_{0.25}\left(x_{6}\right)$ & $0.476^{* *}$ & & & $\operatorname{AM}\left(x_{23}\right)$ & $0.519^{* *}$ \\
\hline $\mathrm{WR}_{0.25}\left(x_{7}\right)$ & $0.606^{* *}$ & & & $\mathrm{P}\left(x_{24}\right)$ & $0.759^{* *}$ \\
\hline D-MWD $\left(x_{8}\right)$ & $0.343^{*}$ & & & $\mathrm{H}\left(x_{25}\right)$ & $0.594^{* *}$ \\
\hline W-MWD & 0.327 & & & $\mathrm{D}\left(x_{26}\right)$ & $0.580^{* *}$ \\
\hline D-GMD $\left(x_{9}\right)$ & $0.429^{* *}$ & & & $\mathrm{E}\left(x_{27}\right)$ & $0.590^{* *}$ \\
\hline W-GMD $\left(x_{10}\right)$ & $0.570^{\star *}$ & & & & \\
\hline
\end{tabular}

Table 2. Correlation coefficients of soil environment factors and rapeseed yield. ${ }^{\star}$ Significant at the 0.05 probability level. ${ }^{*}$ Significant at the 0.01 probability level.

\begin{tabular}{|c|c|c|c|c|c|c|c|c|c|c|c|c|c|c|}
\hline Index & N100B0 & N100B10 & N100B20 & N100B40 & N80 B0 & N80 B10 & N80 B20 & N80 B40 & N60 B0 & N60 B10 & N60 B20 & N60 B40 & $\gamma_{\mathrm{i}}$ & $w_{\mathrm{i}}$ \\
\hline$x_{1}$ & 0.767 & 0.997 & 0.982 & 0.982 & 0.647 & 0.898 & 0.750 & 0.739 & 0.580 & 0.610 & 0.524 & 0.495 & 0.748 & 0.035 \\
\hline$x_{2}$ & 0.858 & 1.000 & 0.933 & 0.931 & 0.717 & 0.998 & 0.833 & 0.783 & 0.707 & 0.694 & 0.574 & 0.531 & 0.796 & 0.038 \\
\hline$x_{3}$ & 0.744 & 1.000 & 0.963 & 0.929 & 0.630 & 0.941 & 0.745 & 0.705 & 0.559 & 0.637 & 0.530 & 0.484 & 0.739 & 0.035 \\
\hline$x_{4}$ & 0.942 & 1.000 & 0.959 & 0.868 & 0.993 & 0.993 & 0.913 & 0.865 & 0.875 & 0.710 & 0.651 & 0.681 & 0.871 & 0.041 \\
\hline$x_{5}$ & 0.838 & 1.000 & 0.920 & 0.953 & 0.685 & 0.940 & 0.807 & 0.758 & 0.618 & 0.644 & 0.547 & 0.504 & 0.768 & 0.036 \\
\hline$x_{6}$ & 0.731 & 0.999 & 0.993 & 0.967 & 0.617 & 0.890 & 0.752 & 0.715 & 0.555 & 0.613 & 0.545 & 0.498 & 0.740 & 0.035 \\
\hline$x_{7}$ & 0.900 & 1.000 & 0.891 & 0.892 & 0.735 & 0.913 & 0.828 & 0.849 & 0.642 & 0.697 & 0.602 & 0.547 & 0.791 & 0.037 \\
\hline$x_{8}$ & 0.712 & 1.000 & 0.970 & 0.963 & 0.620 & 0.910 & 0.747 & 0.720 & 0.539 & 0.612 & 0.537 & 0.494 & 0.735 & 0.035 \\
\hline$x_{9}$ & 0.835 & 1.000 & 0.793 & 0.820 & 0.716 & 0.919 & 0.917 & 0.891 & 0.623 & 0.618 & 0.654 & 0.597 & 0.782 & 0.037 \\
\hline$x_{10}$ & 0.770 & 1.000 & 0.725 & 0.745 & 0.964 & 0.959 & 0.999 & 0.922 & 0.884 & 0.862 & 0.720 & 0.625 & 0.848 & 0.040 \\
\hline$x_{11}$ & 0.862 & 1.000 & 0.961 & 0.784 & 0.714 & 0.980 & 0.766 & 0.872 & 0.649 & 0.759 & 0.641 & 0.676 & 0.805 & \begin{tabular}{|l|}
0.038 \\
\end{tabular} \\
\hline$x_{12}$ & 0.812 & 1.000 & 0.780 & 0.782 & 0.788 & 0.793 & 0.989 & 0.871 & 0.751 & 0.836 & 0.670 & 0.691 & 0.814 & \begin{tabular}{|l|}
0.038 \\
\end{tabular} \\
\hline$x_{13}$ & 0.721 & 1.000 & 0.954 & 0.953 & 0.637 & 0.987 & 0.840 & 0.797 & 0.580 & 0.669 & 0.590 & 0.542 & 0.773 & 0.037 \\
\hline$x_{14}$ & 0.849 & 1.000 & 0.913 & 0.822 & 0.810 & 0.719 & 0.786 & 0.776 & 0.950 & 0.953 & 0.838 & 0.859 & 0.856 & 0.040 \\
\hline$x_{15}$ & 0.936 & 0.949 & 0.824 & 0.675 & 0.989 & 0.890 & 0.903 & 0.895 & 0.837 & 0.617 & 0.684 & 0.757 & 0.830 & 0.039 \\
\hline$x_{16}$ & 0.942 & 0.850 & \begin{tabular}{|l|l}
0.778 \\
\end{tabular} & 0.657 & 0.952 & 0.890 & 0.791 & 0.837 & 0.910 & 0.782 & 0.756 & 0.892 & 0.837 & 0.040 \\
\hline$x_{17}$ & 0.376 & 1.000 & 0.339 & 0.333 & 0.403 & 0.932 & 0.374 & 0.368 & 0.440 & 0.789 & 0.459 & 0.479 & 0.524 & 0.025 \\
\hline$x_{18}$ & 0.971 & 0.775 & 0.678 & 0.646 & 0.990 & 0.890 & 0.949 & 0.741 & 0.875 & 0.799 & 0.742 & 0.844 & 0.825 & 0.039 \\
\hline$x_{19}$ & 0.959 & 0.999 & 0.906 & 0.956 & 0.865 & 0.946 & 0.977 & 0.932 & 0.740 & 0.720 & 0.632 & 0.591 & 0.852 & \begin{tabular}{|l|}
0.040 \\
\end{tabular} \\
\hline$x_{20}$ & 0.942 & 1.000 & 0.916 & 0.761 & 0.909 & 0.873 & 0.993 & 0.942 & 0.771 & 0.763 & 0.641 & 0.633 & 0.845 & 0.040 \\
\hline$x_{21}$ & 0.761 & 1.000 & 0.932 & 0.971 & 0.659 & 0.916 & 0.772 & 0.798 & 0.622 & 0.663 & 0.548 & 0.544 & 0.766 & 0.036 \\
\hline$x_{22}$ & 0.907 & 1.000 & 0.917 & 0.768 & 0.976 & 0.932 & 0.951 & 0.944 & 0.812 & 0.743 & 0.635 & 0.625 & 0.851 & 0.040 \\
\hline$x_{23}$ & 0.863 & 0.980 & 0.906 & 0.825 & 0.799 & 0.874 & 0.915 & 0.982 & 0.679 & 0.761 & 0.609 & 0.577 & 0.814 & \begin{tabular}{|l|}
0.038 \\
\end{tabular} \\
\hline$x_{24}$ & 0.771 & 1.000 & 0.925 & 0.644 & 0.767 & 0.938 & 0.885 & 0.843 & 0.880 & 0.893 & 0.836 & 0.759 & 0.845 & \begin{tabular}{|l|}
0.040 \\
\end{tabular} \\
\hline$x_{25}$ & 0.691 & 0.998 & 0.908 & 0.888 & 0.589 & 0.890 & $\begin{array}{l}0.699 \\
\end{array}$ & 0.667 & 0.525 & 0.613 & 0.504 & 0.464 & 0.703 & 0.033 \\
\hline$x_{26}$ & 0.683 & 1.000 & 0.907 & 0.870 & 0.582 & 0.890 & 0.694 & 0.659 & 0.519 & 0.610 & 0.500 & 0.458 & 0.698 & 0.033 \\
\hline$x_{27}$ & 0.691 & 0.998 & 0.908 & 0.888 & 0.589 & 0.890 & 0.699 & 0.667 & 0.525 & 0.613 & 0.504 & 0.464 & 0.703 & 0.033 \\
\hline
\end{tabular}

Table 3. Correlation coefficient.

Comprehensive evaluation and analysis of soil fertilization. In order to evaluate the fertilization effect of each treatment intuitively and accurately, the corresponding $\mathrm{Y}$ value was calculated according to the feature vector and the standardized value: $\mathrm{Y} 1=0.197 x_{1}+0.176 x_{2}+0.203 x_{3}+0.210 x_{4}+0.186 x_{5}+0.187 x_{6}+0.208 x_{7}+0.160 x_{8}+0.1$ $80 x_{9}+0.191 x_{10}+0.181 x_{11}+0.176 x_{12}+0.163 x_{13}+0.176 x_{14}+0.206 x_{15}+0.201 x_{16}+0.187 x_{17}+0.188 x_{18}+0.195 x_{19}+0$. $201 x_{20}+0.206 x_{21}+0.203 x_{22}+0.187 x_{23}+0.204 x_{24}+0.206 x_{25}+0.204 x_{26}+0.205 x_{27}$. Similarly, Y2 and Y3 were also calculated. The weighted mean was obtained by taking the contribution rate of the three principal components as 


\begin{tabular}{|l|l|l|l|l|}
\hline Treatments & Grey comprehensive evaluation value & Comprehensive ranking & Yield value & Yield ranking \\
\hline N100B0 & 0.817 & 6 & 2.11 & 6 \\
\hline N100B10 & 0.980 & 1 & 2.57 & 1 \\
\hline N100B20 & 0.877 & 3 & 2.47 & 2 \\
\hline N100B40 & 0.825 & 5 & 2.42 & 4 \\
\hline N80B0 & 0.764 & 8 & 1.86 & 9 \\
\hline N80B10 & 0.909 & 2 & 2.45 & 3 \\
\hline N80B20 & 0.834 & 4 & 2.13 & 5 \\
\hline N80B40 & 0.806 & 7 & 2.05 & 7 \\
\hline N60B0 & 0.700 & 10 & 1.65 & 10 \\
\hline N60B10 & 0.716 & 9 & 1.94 & 8 \\
\hline N60B20 & 0.623 & 11 & 1.58 & 11 \\
\hline N60B40 & 0.611 & 12 & 1.40 & 12 \\
\hline
\end{tabular}

Table 4. Grey judgement analysis of soil fertilization in different treatments.

\begin{tabular}{|c|c|c|c|c|c|c|c|c|c|c|c|c|c|c|c|c|c|c|c|c|c|c|c|c|c|c|c|}
\hline & $x_{1}$ & $x_{2}$ & $x_{3}$ & $x_{4}$ & $x_{5}$ & $x_{6}$ & $x_{7}$ & $x_{8}$ & $x_{9}$ & $x_{10}$ & $x_{11}$ & $x_{12}$ & $x_{13}$ & $x_{14}$ & $x_{15}$ & $x_{16}$ & $x_{17}$ & $x_{18}$ & $x_{19}$ & $x_{20}$ & $x_{21}$ & $x_{22}$ & $x_{23}$ & $x_{24}$ & $x_{25}$ & $x_{26}$ & $x_{27}$ \\
\hline$x_{1}$ & - & * & ** & ns & ns & * & $* *$ & * & * & $* *$ & ** & ns & \begin{tabular}{|l|l|} 
ns \\
\end{tabular} & ns & ns & * & $* *$ & ns & * & * & \begin{tabular}{|l|l|} 
ns \\
\end{tabular} & $* *$ & ns & ** & ** & * & $*$ \\
\hline$x_{2}$ & & - & ** & ns & ** & ns & $* *$ & \begin{tabular}{|l|l|} 
ns \\
\end{tabular} & ns & $* *$ & ns & * & ns & $* *$ & ns & ns & * & ns & ** & ** & ** & $* *$ & ns & ** & $* *$ & *** & $* *$ \\
\hline$x_{3}$ & & & - & ns & * & ns & ns & ns & ns & ns & ns & ns & ns & ns & ns & ns & ns & ns & * & ns & * & * & ns & * & ns & ns & ns \\
\hline$x_{4}$ & & & & - & * & * & $* *$ & \begin{tabular}{|l|} 
ns \\
\end{tabular} & $* *$ & *** & ns & ** & * & $* *$ & * & $* *$ & $* *$ & ns & ** & ** & * & $* *$ & ** & ** & $* *$ & ** & ** \\
\hline$x_{5}$ & & & & & - & * & $* *$ & \begin{tabular}{|l|} 
ns \\
\end{tabular} & * & ** & ns & * & \begin{tabular}{|l|} 
ns \\
\end{tabular} & ns & ns & $* * *$ & $* *$ & ns & ** & ** & ** & $* *$ & * & ** & ** & ** & ** \\
\hline$x_{6}$ & & & & & & - & $* *$ & ** & ** & *** & * & * & * & * & $* *$ & $* *$ & $* *$ & ** & *** & *** & * & $* *$ & * & *** & $* *$ & ** & ** \\
\hline$x_{7}$ & & & & & & & - & ** & $* *$ & ** & ** & ** & \begin{tabular}{|l|} 
ns \\
\end{tabular} & $* *$ & $* *$ & $* *$ & $* *$ & ** & ** & $* *$ & ** & $* *$ & ** & ** & $* *$ & ** & ** \\
\hline$x_{8}$ & & & & & & & & - & $* *$ & ns & ns & $* *$ & ** & * & ns & * & ** & ns & ns & ns & * & * & ns & * & $* *$ & *** & *** \\
\hline$x_{9}$ & & & & & & & & & - & ** & * & $* *$ & * & * & $* *$ & $* *$ & $* *$ & ** & * & ** & * & ** & * & ** & ** & ** & ** \\
\hline$x_{10}$ & & & & & & & & & & - & * & * & ns & $* *$ & $* *$ & * & $* *$ & * & $* *$ & $* *$ & * & $* *$ & ** & ** & $* *$ & ** & ** \\
\hline$x_{11}$ & & & & & & & & & & & - & ns & ns & $* *$ & ** & $* *$ & * & * & * & * & \begin{tabular}{|l|} 
ns \\
\end{tabular} & $* *$ & * & * & * & ** & * \\
\hline$x_{12}$ & & & & & & & & & & & & - & *** & $* *$ & ns & ns & * & ns & *** & *** & ** & $* *$ & ** & *** & ** & *** & *** \\
\hline$x_{13}$ & & & & & & & & & & & & & - & $* *$ & ns & * & $* *$ & ns & *** & ** & ns & * & * & * & * & *** & * \\
\hline$x_{14}$ & & & & & & & & & & & & & & - & * & ** & $* *$ & \begin{tabular}{|l|l} 
ns \\
\end{tabular} & $* *$ & ** & ** & $* *$ & ** & ** & ** & ** & ** \\
\hline$x_{15}$ & & & & & & & & & & & & & & & - & ** & $* *$ & ** & $* *$ & ** & ns & $* *$ & * & $* *$ & ** & ** & $* *$ \\
\hline$x_{16}$ & & & & & & & & & & & & & & & & - & ** & ** & *** & *** & * & $* *$ & ** & ** & $* *$ & *** & *** \\
\hline$x_{17}$ & & & & & & & & & & & & & & & & & - & ** & ** & ** & ** & $* *$ & * & ** & $* *$ & ** & ** \\
\hline$x_{18}$ & & & & & & & & & & & & & & & & & & - & ns & $*$ & ns & * & ns & ** & * & ** & * \\
\hline$x_{19}$ & & & & & & & & & & & & & & & & & & & - & ** & ** & $* *$ & ** & ** & $* *$ & ** & ** \\
\hline$x_{20}$ & & & & & & & & & & & & & & & & & & & & - & ** & $* *$ & ** & ** & $* *$ & ** & ** \\
\hline$x_{21}$ & & & & & & & & & & & & & & & & & & & & & - & $* *$ & ** & ** & $* *$ & ** & ** \\
\hline$x_{22}$ & & & & & & & & & & & & & & & & & & & & & & - & ** & ** & $* *$ & ** & ** \\
\hline$x_{23}$ & & & & & & & & & & & & & & & & & & & & & & & - & ** & ** & ** & $* *$ \\
\hline$x_{24}$ & & & & & & & & & & & & & & & & & & & & & & & & - & $* *$ & ** & ${ }^{* *}$ \\
\hline$x_{25}$ & & & & & & & & & & & & & & & & & & & & & & & & & - & ** & $* *$ \\
\hline$x_{26}$ & & & & & & & & & & & & & & & & & & & & & & & & & & - & *** \\
\hline$x_{27}$ & & & & & & & & & & & & & & & & & & & & & & & & & & & - \\
\hline
\end{tabular}

Table 5. Correlation analysis. ${ }^{*}$ Significant at the 0.05 probability level. ${ }^{*}$ Significant at the 0.01 probability level; ns Not significant.

\begin{tabular}{|l|l|l|l|}
\hline Principal component & Eigenvalue & Contribution rate (\%) & Accumulative contribution rate (\%) \\
\hline 1 & 20.496 & 75.911 & 75.911 \\
\hline 2 & 2.487 & 9.211 & 85.122 \\
\hline 3 & 1.708 & 6.327 & 91.448 \\
\hline
\end{tabular}

Table 6. Eigenvalues and cumulative contribution proportions of principle components of the indices. 


\begin{tabular}{|l|l|r|r|}
\hline Index & Prin1 & Prin2 & Prin3 \\
\hline$x_{1}$ & 0.197 & 0.053 & -0.285 \\
\hline$x_{2}$ & 0.176 & -0.307 & -0.093 \\
\hline$x_{3}$ & 0.203 & -0.114 & -0.133 \\
\hline$x_{4}$ & 0.210 & -0.129 & -0.056 \\
\hline$x_{5}$ & 0.186 & 0.019 & -0.371 \\
\hline$x_{6}$ & 0.187 & 0.306 & -0.033 \\
\hline$x_{7}$ & 0.208 & 0.047 & -0.168 \\
\hline$x_{8}$ & 0.160 & 0.409 & 0.047 \\
\hline$x_{9}$ & 0.180 & 0.349 & -0.018 \\
\hline$x_{10}$ & 0.191 & -0.036 & -0.286 \\
\hline$x_{11}$ & 0.181 & 0.060 & 0.298 \\
\hline$x_{12}$ & 0.176 & 0.048 & 0.362 \\
\hline$x_{13}$ & 0.163 & 0.189 & 0.393 \\
\hline$x_{14}$ & 0.176 & -0.193 & 0.294 \\
\hline$x_{15}$ & 0.206 & 0.123 & -0.112 \\
\hline$x_{16}$ & 0.201 & 0.093 & 0.065 \\
\hline$x_{17}$ & 0.187 & 0.254 & -0.233 \\
\hline$x_{18}$ & 0.188 & 0.216 & 0.056 \\
\hline$x_{19}$ & 0.195 & -0.254 & -0.017 \\
\hline$x_{20}$ & 0.201 & -0.223 & 0.021 \\
\hline$x_{21}$ & 0.206 & -0.096 & 0.140 \\
\hline$x_{22}$ & 0.203 & -0.222 & -0.077 \\
\hline$x_{23}$ & 0.187 & -0.249 & 0.191 \\
\hline$x_{24}$ & 0.204 & -0.198 & -0.057 \\
\hline$x_{25}$ & 0.206 & -0.007 & 0.010 \\
\hline$x_{26}$ & 0.204 & 0.006 & 0.191 \\
\hline$x_{27}$ & 0.205 & 0.001 & 0.009 \\
\hline & & & \\
\hline
\end{tabular}

Table 7. Eigenvalues of the indices under the three principal components.

\begin{tabular}{|l|l|l|l|l|}
\hline Treatments & Comprehensive value & Comprehensive ranking & Yield value & Yield ranking \\
\hline N100B0 & -0.326 & 6 & 2.11 & 6 \\
\hline N100B10 & 6.519 & 1 & 2.57 & 1 \\
\hline N100B20 & 2.683 & 3 & 2.47 & 2 \\
\hline N100B40 & -0.817 & 7 & 2.42 & 4 \\
\hline N80B0 & -2.541 & 9 & 1.86 & 9 \\
\hline N80B10 & 4.692 & 2 & 2.45 & 3 \\
\hline N80B20 & 0.053 & 5 & 2.13 & 5 \\
\hline N80B40 & -2.802 & 10 & 2.05 & 7 \\
\hline N60B0 & -3.547 & 11 & 1.65 & 10 \\
\hline N60B10 & 2.540 & 4 & 1.94 & 8 \\
\hline N60B20 & -1.893 & 8 & 1.58 & 11 \\
\hline N60B40 & -4.560 & 12 & 1.40 & 12 \\
\hline
\end{tabular}

Table 8. Principle analysis of soil fertilization in different treatments.

the weight, and the composite score of principal component $\mathrm{Y}=75.911 \% \mathrm{Y} 1+9.211 \% \mathrm{Y} 2+6.327 \% \mathrm{Y}$. The comprehensive score could be used as a comprehensive evaluation index for the effect of biochar combined with nitrogen reduction on soil fertility (Table 8). It could be seen from Table 8 , the change trend of soil fertilization in each treatment was consistent with that of yield. Under the same nitrogen application rate, the comprehensive score of B10 treatment was greater than that of B0, B20, and B40 treatment; under the same biochar, the comprehensive score of N60 and N80 treatment was less than that of N100 treatment. Among all the treatments, N100B10 had the highest comprehensive score, followed by N80B10, and N60B40 was lowest. It indicated that B10 was conducive to soil fertilization and crop yield increase, and the combined with N80 could achieve nitrogen reduction and efficiency increase, while nitrogen reduction (N60) was not conducive to soil fertilization and crop yield increase. 


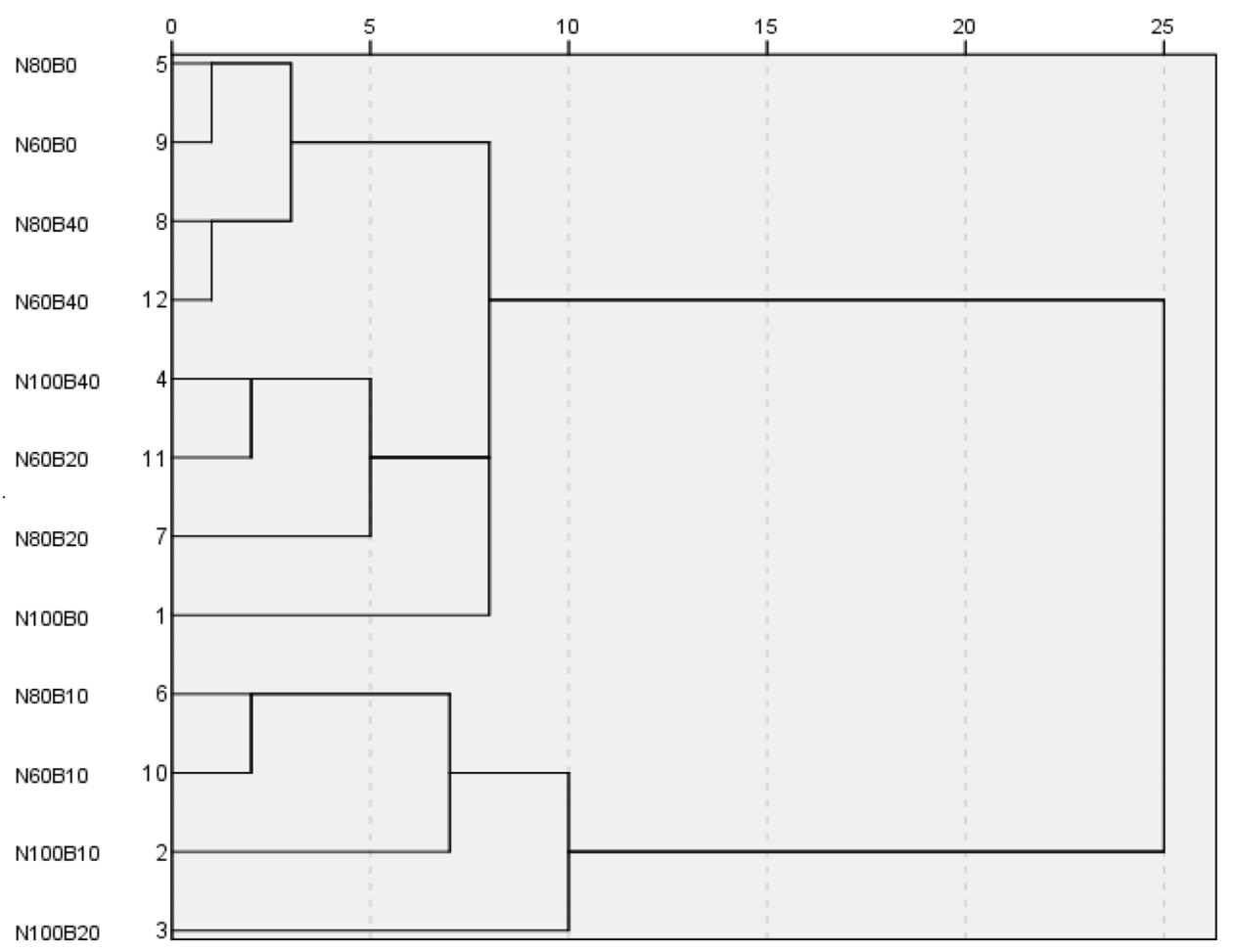

Figure 1. Cluster analysis of soil fertilization in each treatment.

Cluster analysis. As shown in Fig. 1, if the distance threshold was set to 8, the systematic clustering according to the degree of intimacy and similarity of the soil fertility level could well reflect the effect of biochar combined with nitrogen reduction on soil fertility. In short, the 12 treatments could be roughly divided into 5 categories. In other words, N80B0, N80B40, N60B0 and N60B40 belonged to a category, which were considered a low fertility level; N100B10, N80B10 and N60B10 belonged to a category, which were considered a high fertility level; N100B40, N60B20 and N80B20 belonged to a category, and N100B0 and N100B20 was each a category, which were considered a medium fertility levels. It showed that biochar and nitrogen fertilizer could affect the soil fertility level in different degrees.

\section{Discussion}

Improving soil fertility requires not only building a good soil structure, but also cultivating fertile farming layers to improve soil productivity. Therefore, the evaluation of soil fertilization in each treatment must be comprehensively reflected from the perspectives of soil physics, chemistry and biology ${ }^{32}$. In this study, 27 indicators being significantly correlated with yield were selected as evaluation indicators through correlation analysis. Correlation analysis showed that there were different degrees of correlation between soil physical, chemical and biological indicators, indicating that they were connected and interact with each other, and they jointly affected the soil fertilization effect, which was similar to the results of Yuan et al. ${ }^{33}$. However, the selection of evaluation indicators and evaluation methods is slightly different due to different test purposes and there is no uniform evaluation standard and fixed method in the world, but evaluation indicators generally cover soil physical, chemical and biological indicators and evaluation methods generally include grey correlation analysis, path analysis, principal component analysis, factor analysis and cluster analysis. In our experiment, gray correlation analysis and principal component analysis showed that the comprehensive evaluation value and comprehensive score were basically consistent with rapeseed yield. Generally, the features of soil quality can often be directly and comprehensively displayed through crop yields, making the comprehensive evaluation results more accurate, objective and scientific ${ }^{34}$. Therefore, the selected indicators and methods in this study were reliable for evaluating soil fertility status.

Our study results indicated that biochar combined with nitrogen reduction mainly changed AP and W-GMD in soil physical indexes, URE in soil chemical indicators, and F, S, AA, P and CA in soil biological indexes. This may be related to the local low-phosphorus and arid environment. Currently, there are few studies on the evaluation of soil quality under biochar combined with nitrogen reduction, and further research verification are needed. From the feature vector of the first principal component of principal component analysis, it could be seen that biochar combined with nitrogen reduction mainly changed AP and W-GMD in soil physical indicators, DOC in soil chemical indicators, and B, S, AA, PA, P, CA, H, D and E in soil biological indicators. Principal component 1 explained $75.911 \%$ of the difference, which basically reflected the information provided by all soil indicators, making the evaluation more scientific and reasonable. Therefore, we summarized that the available phosphorus, the geometric mean diameter of water stability, fungi number, the utilization degree of microorganisms on sugars, 
amino acids, polymers and carboxylic acids could be used as the main soil factors affecting rapeseed yield under biochar combined with nitrogen reduction.

Cluster analysis showed that no biochar, nitrogen fertilizer reduction and high biochar resulted in low soil fertility levels, while appropriate biochar, moderate nitrogen fertilizer, and biochar combined with nitrogen reduction brought about high soil fertility levels. This indicated that appropriate amount of biochar and nitrogen fertilizer was beneficial to the improvement of soil fertility, being similar to reports by Nasim et al. ${ }^{35}$ and Veysel et al. ${ }^{36}$. This was mainly because biochar itself provides nutrients and retains nutrients, and changes the kinetics of soil microorganisms, thus promoting biological carbon fixation ${ }^{37}$. As for nitrogen fertilizer, it is a kind of quick-acting nitrogen, which is beneficial to the improvement of soil effective nutrients after applied to the soil ${ }^{38}$. Generally, the yield can reflect the soil fertility to a certain extent. In this study, rapeseed yields of N100B10, N100B20 and N80B10 were the highest, followed by other treatments at the N100 and B10 levels, while the yields at the N60, B40 and B0 levels were the lowest, being basically consistent with the results of cluster analysis. It was feasible to use cluster analysis to classify the soil fertility level, which was in line with objective reality, and could be used as a basis for evaluating the effect of biochar combined with nitrogen reduction on soil fertility.

\section{Conclusions}

Available phosphorus, geometric mean diameter of water stability, fungi number, utilization of microorganisms on sugars, amino acids, polymers and carboxylic acids were the main soil factors affecting soil fertilization and rapeseed yield under biochar combined with nitrogen reduction based on grey correlation analysis and principal component analysis. Besides, based on grey correlation analysis, principal component analysis and cluster analysis, the combined application of $10 \mathrm{t} \mathrm{hm}^{-2}$ biochar and 144 or $180 \mathrm{~kg} \mathrm{hm}^{-2}$ nitrogen fertilizers had better fertilization effect. From the perspective of comprehensive economic and environmental benefits, $10 \mathrm{t} \mathrm{hm}^{-2}$ biochar combined with $144 \mathrm{~kg} \mathrm{hm}^{-2}$ nitrogen fertilizer was the optimal fertilization model in uplands in purple soil area of southwest China.

Received: 14 December 2020; Accepted: 14 June 2021

Published online: 02 July 2021

\section{References}

1. Mulugeta, D. \& Karl, S. Assessment of integrated soil and water conservation measures on key soil properties in south Gondar, north-western Highlands of Ethiopia. J. Soil Sci. Environ. Manage 1, 164-176 (2010).

2. Kahrl, F., Yunju, L., Roland-Holst, D., Jianchu, X. \& Zilberman, D. Toward sustainable use of nitrogen fertilizers in China (2012).

3. Tian, D. S. \& Niu, S. L. A global analysis of soil acidification caused by nitrogen addition. Environ. Res. Lett. 2, 204019 (2015).

4. Qu, Z., Wang, J., Almøy, T. \& Bakken, L. R. Excessive use of nitrogen in Chinese agriculture results in high $\mathrm{N}_{2} \mathrm{O} /\left(\mathrm{N}_{2} \mathrm{O}+\mathrm{N}_{2}\right)$ product ratio of denitrification, primarily due to acidification of the soils. Global Change Biol. 20, 1685-1698 (2014).

5. Wang, D., Xu, Z. Z., Zhao, J. Y., Wang, Y. F. \& Yu, Z. W. Excessive nitrogen application decreases grain yield and increases nitrogen loss in a wheat-soil system. Acta Agric. Scand. Sect, B-S. P. 61, 681-692 (2011).

6. Lan, T. et al. Regulating $\mathrm{CH}_{4}, \mathrm{~N}_{2} \mathrm{O}$, and $\mathrm{NO}$ emissions from an alkaline paddy field under rice-wheat rotation with controlled release N fertilizer. Environ. Sci. Pollut. R. 28, 18246-18259 (2021).

7. Weber, K. \& Quicker, P. Properties of biochar. Fuel 217, 240-261 (2018).

8. Guo, X. X., Liu, H. T. \& Zhang, J. The role of biochar in organic waste composting and soil improvement: A review. Waste Manage 102, 884-899 (2020).

9. Muhammad, Z. et al. Efficacy of chitosan-coated textile waste biochar applied to Cd-polluted soil for reducing Cd mobility in soil and its distribution in moringa (Moringa oleifera L.). J. Environ. Manage. 284, 112047 (2021).

10. Veysel, T. Confident performance of chitosan and pistachio shell biochar on reducing Ni bioavailability in soil and plant plus improved the soil enzymatic activities, antioxidant defense system and nutritional quality of lettuce. Ecotox. Environ. Safe. 183, 109594 (2019).

11. Liu, D. et al. Biochar and compost enhance soil quality and growth of roselle (Hibiscus sabdariffa L.) under saline conditions. Sci. Rep. 11, 1 (2021).

12. Wang, F. L., Wang, X. X. \& Song, N. N. Biochar and vermicompost improve the soil properties and the yield and quality of cucumber (Cucumis sativus L.) grown in plastic shed soil continuously cropped for different years. Agr. Ecosyst. Environ. 315, 107425 (2021).

13. Nabilla, S. A., Anni, Y., Ade, S. \& Yuliati, M. The effect of N, P, K fertilizer and Nano Silica fertilizer to total N content, N uptake, and black rice yield (Oryza sativa L. Indica) on inceptisols from jatinangor. J. Plant Sci. 8, 185 (2020).

14. Peng, H. H. Effects of biochar, organic fertilizer and chemical fertilizer combined application on nutrient utilization and yield of spring maize. J. South Agric. 46, 1396 (2015).

15. Singh, R., Singh, P., Singh, H. \& Raghubanshi, A. S. Impact of sole and combined application of biochar, organic and chemical fertilizers on wheat crop yield and water productivity in a dry tropical agro-ecosystem. Biochar 1, 229 (2019).

16. Shrestha, A. J. \& Pandit, B. H. Action research into a flood resilient value chain- biochar-based organic fertilizer replaces chemical fertilizer with double yield of pea in Udayapur, Nepal. Am. J. Agric. For. 5, 84-93 (2017).

17. Peng, J., Han, X. R., Li, N., Chen, K., Yang, J. F., Zhan, X. M., Luo, P. Y. \& Liu, N. Combined application of biochar with fertilizer promotes nitrogen uptake in maize by increasing nitrogen retention in soil. Biochar 3, 1-13 (2021).

18. Khan, Z. et al. Coupling of biochar with nitrogen supplements improve soil fertility, nitrogen utilization efficiency and rapeseed growth. Agronomy 10, 1661-1661 (2020).

19. Zahra, M. B., Aftab, Z. E. H., Akhter, A. \& Haider, M. S. Cumulative effect of biochar and compost on nutritional profile of soil and maize productivity. J. Plant Nutr. 44, 1664-1676 (2021).

20. Kalu, S., Simojoki, A., Karhu, K. \& Tammeorg, P. Long-term effects of softwood biochar on soil physical properties, greenhouse gas emissions and crop nutrient uptake in two contrasting boreal soils. Agric. Ecosyst. Environ. 316, 107454 (2021).

21. Zhong, S. Q. et al. Relationships between the lithology of purple rocks and the pedogenesis of purple soils in the Sichuan Basin, china. Sci. Rep. 9, 1-13 (2019).

22. Sui, Y. Y., Zhang, X. Y., Gu, S. Y. \& Chen, X. Study of evaluation systems of the black soil fertility. Syst. Sci. Comp. Stud. Agric. 20(4), $265-270(2004)$.

23. Lu, P., Su, Y. R. \& Niu, Z. Soil quality assessment indicators and their spatial temporal variability. Chin. J. Eco-Agric. 15(4), 190-194 (2007).

24. Tian, X. Q., Li, Z., Wang, L. C., Wang, Y. F. \& Li, B. Biochar and slow release urea effects on root morphology, grain yield, nitrogen uptake and utilization in Brassica napus L. Int. J. Agric. Biol. 23, 653-660 (2020). 
25. Menšík, L., Hlisnikovský, L., Pospíšilová, L. \& Kunzová, E. The effect of application of organic manures and mineral fertilizers on the state of soil organic matter and nutrients in the long-term field experiment. J. Soil Sediment 18, 2813-2822 (2018).

26. Wiebe, W. J. \& Smith, D. F. Direct measurement of dissolved organic carbon release by phytoplankton and incorporation by microheterotrophs. Mar. Biol. 42, 213-223 (1977).

27. Wang, Y. et al. The influence of soil heavy metals pollution on soil microbial biomass, enzyme activity, and community composition near a copper smelter. Ecotox. Environ. Safe 67, 75-81 (2007).

28. Zhou, Q., Chen, J., Xing, Y., Xie, X. \& Wang, L. Influence of intercropping Chinese milk vetch on the soil microbial community in rhizosphere of rape. Plant Soil 440, 85-96 (2019).

29. Yeh, Y. L. \& Chen, T. C. Application of grey correlation analysis for evaluating the artificial lake site in Pingtung Plain, Taiwan. Can. J. Civ. Eng. 31, 56-64 (2004).

30. Lever, J., Krzywinski, M. \& Altman, N. Principal component analysis. Nat. Methods 14, 641-642 (2017).

31. Brian, S., Everitt, S. L., Morven, L. \& Daniel, S. Optimization clustering techniques (2011).

32. Fan, H. R., Li, Y. P. \& Wu, S. X. Study on the comprehensive evaluation of the soil fertility quality of the green land in Qinhuangdao. Adv. Mater. Res. 573-574, 191-194 (2012).

33. Yuan, J. J., Tong, Y. A., Lu, S. H. \& Yuan, G. J. Comprehensive evaluation on soil fertility quality of jujube orchard under combined application of biochar and nitrogen fertilizer. Trans. CSAE 34, 134-140 (2018).

34. Meena, V. D. et al. A case for silicon fertilization to improve crop yields in tropical soils. Proc. Natl. Acad. Sci. India Sect. B. Biol. Sci 84, 505-518 (2013).

35. Nasim, W. et al. Radiation efficiency and nitrogen fertilizer impacts on sunflower crop in contrasting environments of Punjab, Pakistan. Environ. Sci. Pollut. Res. 25, 1822-1836 (2018).

36. Veysel, T. et al. Promoting the productivity and quality of brinjal aligned with heavy metals immobilization in a wastewater irrigated heavy metal polluted soil with biochar and chitosan. Ecotox. Environ. Safe. 161, 409-419 (2018).

37. Xu, W. et al. Responses of soil microbial biomass, diversity and metabolic activity to biochar applications in managed poplar plantations on reclaimed coastal saline soil. Soil Use Manage. 34(4), 597-605 (2018).

38. Rehab, H. \& Hegab, A. Evaluation of nitrogen sources and polymer coated fertilizers on wheat yield in sandy soil. J. Soil Sci. Plant Nutr. 3(3), 1-12 (2018).

\section{Author contributions}

X.T. wrote the main manuscript text, X.T., L.W. and Z.L. put forward research ideas, and X.T., Y.W. and B.L. collected and analyzed data. All authors reviewed the manuscript.

\section{Funding}

This research was financially supported by the Public Welfare Industry (Agriculture) Research Project (201503127) and National Natural Science Foundation (31871583).

\section{Competing interests}

The authors declare no competing interests.

\section{Additional information}

Correspondence and requests for materials should be addressed to L.W.

Reprints and permissions information is available at www.nature.com/reprints.

Publisher's note Springer Nature remains neutral with regard to jurisdictional claims in published maps and institutional affiliations.

(c) (i) Open Access This article is licensed under a Creative Commons Attribution 4.0 International License, which permits use, sharing, adaptation, distribution and reproduction in any medium or format, as long as you give appropriate credit to the original author(s) and the source, provide a link to the Creative Commons licence, and indicate if changes were made. The images or other third party material in this article are included in the article's Creative Commons licence, unless indicated otherwise in a credit line to the material. If material is not included in the article's Creative Commons licence and your intended use is not permitted by statutory regulation or exceeds the permitted use, you will need to obtain permission directly from the copyright holder. To view a copy of this licence, visit http://creativecommons.org/licenses/by/4.0/.

(C) The Author(s) 2021 